\title{
USING STATISTICAL TOOLS TO DETECT GASOLINE ADULTERATION
}

\author{
Paulo Jorge Sanches Barbeira \\ Departamento de Química- ICEx- UFMG \\ Av. Antonio Carlos, 6627, 31270-901, \\ Belo Horizonte, MG, Brasil \\ barbeira@dedalus.lcc.ufmg.br
}

\begin{abstract}
Histograms and normal distribution curves (Gaussian fit) were used to determine the average composition of the gasoline commercialized in the state of Minas Gerais, Brazil, and to detect samples with atypical composition. Atypical composition may rise from the fact that the gasoline comes from distinct sources (refinery or petrochemical plant) or may be due to the careful addition of industrial solvents. A considerable number of samples of atypical composition have been detected despite the samples tested having the same source. This statistical analytical tool has been shown useful in the evaluation of fuel quality.
\end{abstract}

\section{INTRODUCTION}

The Brazilian fuel market has gone through great changes in last years with the end of the State production and distribution monopoly, which lead to an increased number of fuel dealers, and fuel stations, the opening of the market, a stronger competition, with ensuing great price variation. However, fuel quality has not been guaranteed.

The growing wave of denunciations of adulterated fuel commercialization, specially gasoline, necessitates a fast action by regulating agencies to protect consumers from the harms caused by the bad quality of these products, besides the possible hazards to the environment, and tax evasion resulting from the differentiated taxation of industrial solvents and fuels.

The Fuels Group of the Chemistry Department of UFMG has worked on monitoring gasoline, alcohol and diesel quality, as well as on research projects aiming to develop new analytical methodologies for these petrochemical derivatives.

\section{EXPERIMENTAL}

A total of 1973 samples of regular and special gasoline were collected from July to October 2001 in the east region of Minas Gerais State (Figure 1), in about 560 municipalities and 1800 gas stations.

Twelve parameters were analyzed for each sample by three distinct assays (density, distillation, and infrared spectroscopy): density, temperatures equivalent to 10, 50 and $90 \%$ of the distilled volume, final evaporation point, MON, RON and IAD values, besides benzene, aromatics, olefins and saturated contents.

An automatic densimeter Anton Paar DM 1500 (ASTM D 4052), three automatic distillers Herzog HDA 627 (ASTM D
86), and an infrared analyzer Petrospec GS 1000 were used.

From the number of occurrences as a function of the results obtained in a given assay (histogram), normal distribution graphs (Gaussian) were plotted with their respective average values and standard deviations $(\sigma)$.

Statistically, the results obtained in the range $\pm 3 \sigma$ indicate that the true average value is in this range with a probability of $99,7 \%$. Therefore, the samples in this range present the same behavior (typical samples). Thus, the values outside this confidence interval reflect samples with a behavior different from that of the average, which may be associated to occasional non-conformities when they exceed the values specified by law, or products with distinct characteristics but within specifications.

From July to October 2001, the content of anhydride ethyl alcohol added to gasoline as anti-detonating agent was $22 \pm 1(\% \mathrm{v} / \mathrm{v})$.

\section{RESULTS AND DISCUSSION}

The accumulation of a large number of results obtained for parameters of different assays allowed the use of statistical tools to define the average behavior of commercialized gasoline and to detect occasional differentiated behaviors.

The number of samples with differentiated behavior, atypical samples, is considerable, depending on the parameter analyzed, as shown in Figure 2.

This atypical behavior may have two possible causes: samples with distinct sources (e.g.: distinct refineries), or careful adulteration with the controlled addition of solvents in order to control the product obtained so it falls within the legal specifications in effect. The addition of solvents may lead to engine malfunction, rubber corrosion and environmental 


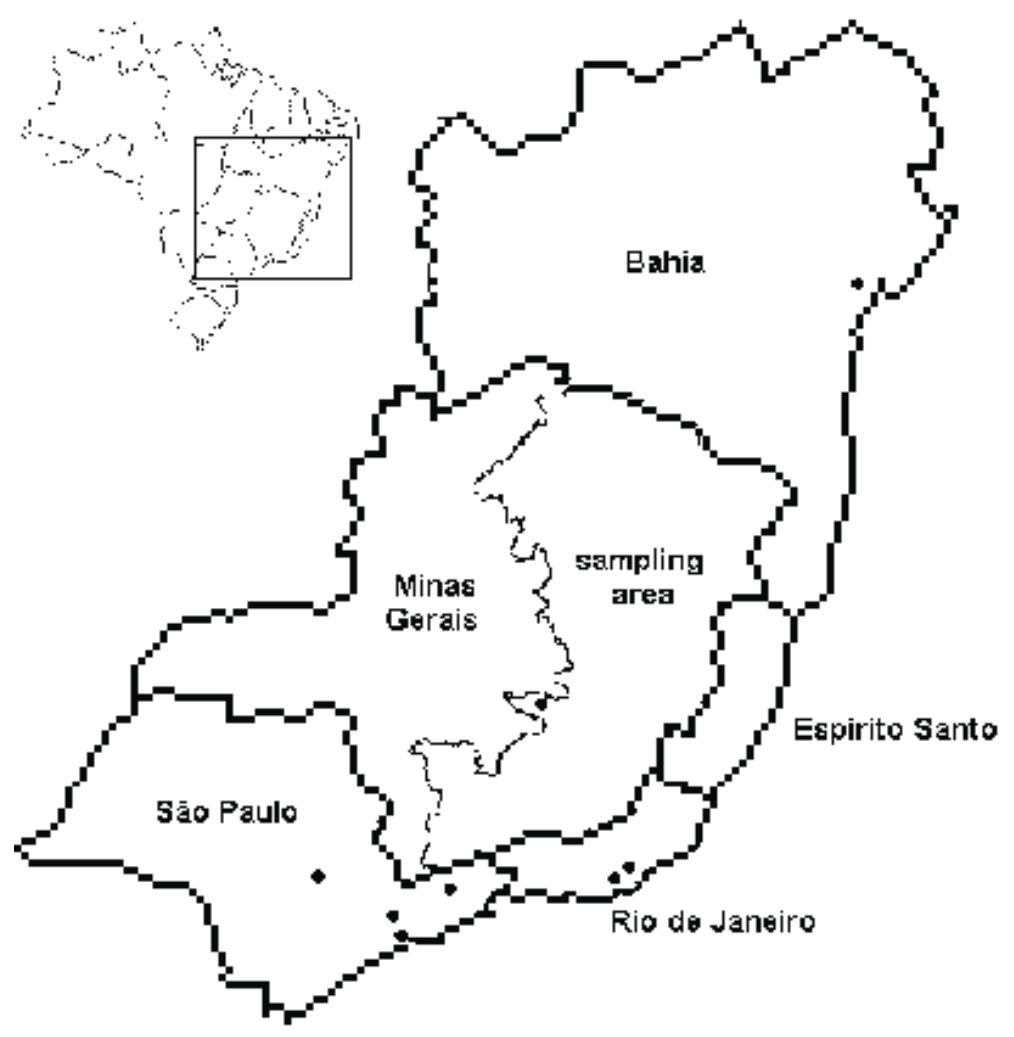

Fig. 1 - Minas Gerais State location, sampling region and neighboring states and location of the closest refineries (black dots).

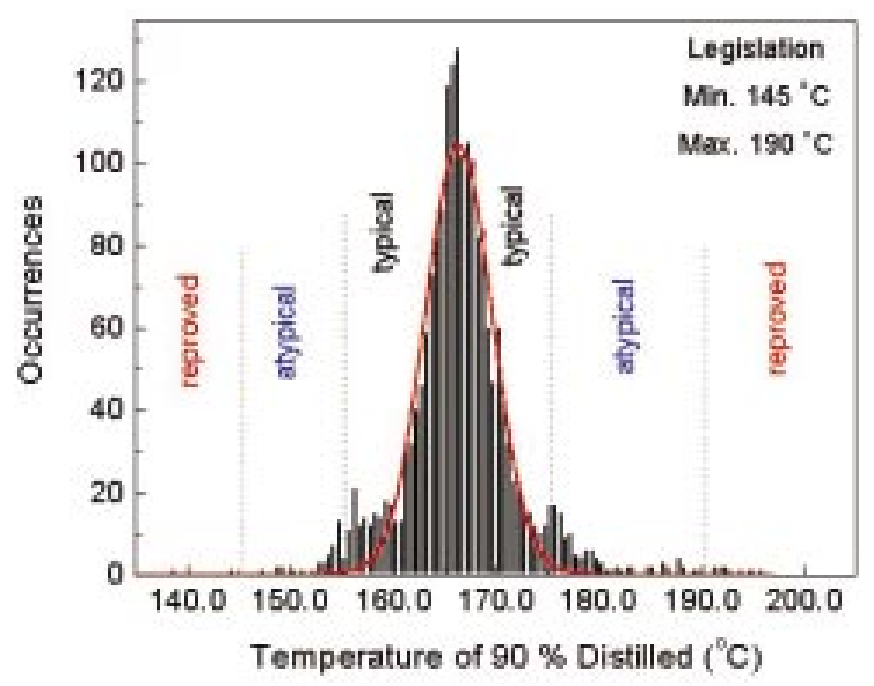

Fig. 2 - Histogram and normal distribution graph for the results obtained for a temperature equivalent to $90 \%$ distilled volume.

hazards, besides tax evasion, since industrial solvents and fuels have different taxation.

In Brazil, refineries and petrochemical plants produce gasoline and sell it distributors, who in turn add ethanol and wholesale it to gas stations. Samples were sorted out according to distributors to distinguish samples with differentiated behavior for having being produced by distinct refineries. In

\begin{tabular}{c|c|c}
\hline $\begin{array}{c}\text { Number of } \\
\text { Atypicalities }\end{array}$ & $\begin{array}{c}\text { Number of } \\
\text { Samples }\end{array}$ & $\mathbf{\%}$ \\
\hline 1 & 81 & 28.1 \\
\hline 2 & 56 & 19.4 \\
\hline 3 & 69 & 24.0 \\
\hline 4 & 44 & 15.3 \\
\hline 5 & 28 & 9.7 \\
\hline 6 & 6 & 2.1 \\
\hline 7 & 3 & 1.0 \\
\hline 8 & 1 & 0.3 \\
\hline
\end{tabular}

Table 1 - Percentual of samples with on or more atypicalities from REGAP.

Minas Gerais State, it was found samples from refineries located in the state itself, São Paulo and Bahia, besides samples whose origin was not possible to establish for lack of information at the retailing gas station.

The profiles of the samples with different origins are quite distinct in relation to some parameters as a reflex of the diverse processes and petrochemical chains used in the refineries. This fact may be observed in histograms and normal distribution graphs for all the samples shown in Figure 3. The two peaks related of the two sample groups are conspicuous, with a confidence interval encompassing the regions of greater incidence of samples. The histograms in Figure $3 \mathrm{~b}$ are for REGAP (I) samples and for São Paulo State samples (II), clearly showing the distinct benzene content of the samples due to distinct production processes and raw materials used.

In this way, 1182 samples were established as originating from Gabriel Passos Refinery (REGAP), located close to the 
Minas Gerais State capital.

The statistical analysis revealed that 288 samples (24\%) presented one or more atypical parameters, as shown in Table I, and $70 \%$ of those had more than one atypical parameter. Atypicalities were detected mainly in density, temperature corresponding to $10 \%$ distilled volume and olefins, aromatics, and saturated contents, which are indicative that these samples probably have a atypical composition due to the careful addition of solvents to these three classes of compounds, thus affecting density and temperature corresponding to $10 \%$ distilled volume, but still allowing their classification as conforming, i.e. in agreement with ANP legal specifications.

The chromatographic study, GC-MS, of these atypical

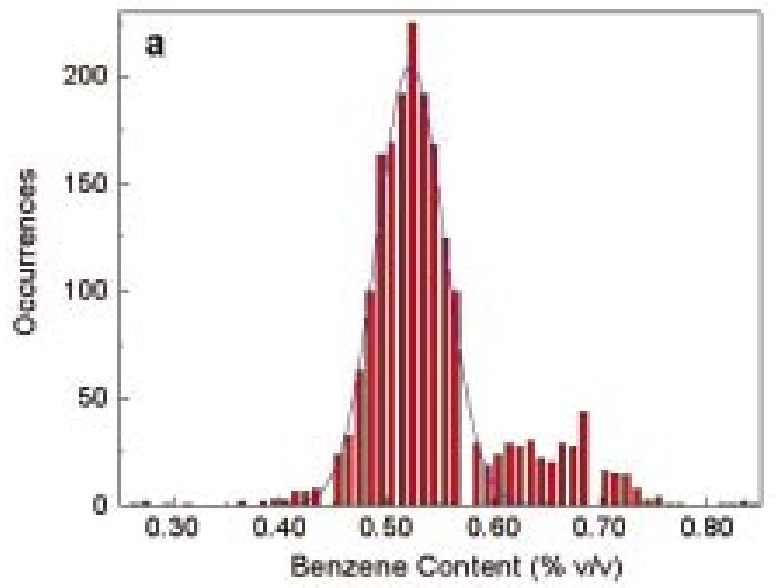

samples is rather useful to determine their characteristics, the possible causes of adulterations, and to identify foreign compounds or differentiated contents in relation to typical samples. The GC-MS analysis in the totality of the samples would be of high time consuming and expensive, due to great number of samples and the necessary time to perform a scan, and a random analysis could not be conclusive.

The determination of confidence intervals has been shown rather useful and fast to determine the average composition of gasoline samples, of distinct regions, and in the detection of samples with differentiated behavior caused by the probable addition of industrial solvents or by distinct origins.

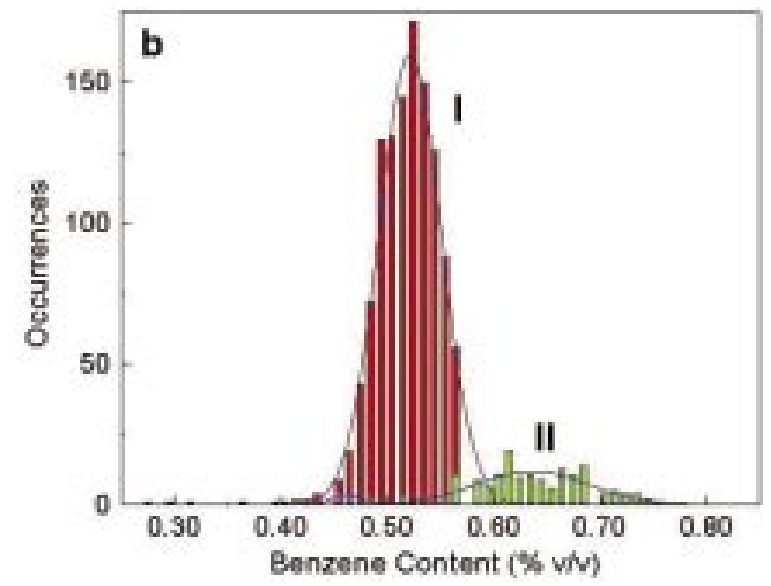

Fig. 3 - Histograms and normal distribution graphs for benzene content results. a) All samples, b) REGAP samples (I), and São Paulo state samples (II). 